\title{
New substituted indene derivatives from bicyclic Baylis-Hillman acetate
}

\author{
Sonia Taktouk, Jihène Ben Kraiem and Hassen Amri * \\ Department of Chemistry, University of Tunis El Manar, Faculty of Science, Campus, 2092 Tunis, Tunisia
}

\begin{abstract}
A convenient protocol for the synthesis of highly functionalized indenes $\mathbf{4}$ has been developed. The coupling reaction of bicyclic Baylis-Hillman acetate $\mathbf{2}$ with nitroalkane salts in basic conditions led to the corresponding substituted indenes in good yields and high purity.
\end{abstract}

Keywords: Bicyclic Baylis-Hillman acetate; nitroalkanes; ethyl 1-alkylidene-1H-indene-2-carboxylates.

\section{Introduction}

The design of new molecules with indene ring is still an open challenge to organic chemists.<smiles>CCC1=C(c2ccc(O)cc2)[C@H](C)c2cc(O)ccc21</smiles>

Indenestrol A Agonist of estrogen receptor
These compounds are important model structures found in various natural products ${ }^{1-3}$ and bioactive compounds ${ }^{4-7}$ (Figure 1).

Figure 1. Some indene skeletons related to various naturel and pharmaceutical products

Various indene-based molecules have shown a wide range of biological activities such as antimicrobial, ${ }^{8}$ anti-inflammatory ${ }^{9}$, aromatase inhibitory ${ }^{10}$, cytotoxic activities ${ }^{11}$, estrogen receptor modulators ${ }^{12}$ and some anti-proliferative activities ${ }^{13}$. In addition, indene derivatives are widely used as building blocks for the synthesis of functional materials ${ }^{14}$ and metallocene complexes for olefin polymerization ${ }^{15}$. Thus, the importance of indenes has stimulated much interest in the construction of indene system including the ring expansion of suitably substituted cyclopropenes, ${ }^{16}$ tandem Friedel Crafts alkylation 17, [3+2] cycloadditions ${ }^{18}, \mathrm{C}-\mathrm{H}$ bond activation ${ }^{19}, \mathrm{C}-\mathrm{C}$ bond cleavage ${ }^{20}$, thermal cascade reactions ${ }^{21}$, ruthenium-mediated ring-closing metathesis ${ }^{22}$, and skeletal rearrangement using some transition metals and Lewis and Brønsted acid catalysts 23-38. However, the above reported approaches suffer from low tolerance of functionality, the use of expensive transition metal catalysts and harsh reaction conditions. Accordingly, the search for an effective and inexpensive method for obtaining substituted indene derivatives is nowadays very sought. Herein, we report an efficient easy twostep synthesis of functionalized indenes $\mathbf{4}$ from the prepared bicyclic Baylis-Hillman acetate $\mathbf{2}$ (Scheme 1).<smiles>[R4]C([R4])=C1C(C(=O)OCC)=Cc2ccccc21</smiles><smiles>CCOC(=O)C1=Cc2ccccc2C1OC(C)=O</smiles><smiles>C=C</smiles><smiles>CCOC(=O)C1=Cc2ccccc2C1O</smiles>

Scheme 1. Retrosynthetic route to highly substituted indenes 4 


\section{Results and Discussion}

Previously, we have described an efficient synthetic method to prepare substituted indenol derivatives $1^{39}$ following the coupling reaction of 1-hydroxy-1H-indene-2-carboxylic acid with alcohols in the presence of $p$-toluenesulfonic acid (PTSA) as a catalyst.Various analogues of indenol derivatives $\mathbf{1}$ have been reported to show a great ability to act as powerful synthons for the synthesis of various useful compounds ${ }^{40,41}$. They were used as templates for the synthesis of various alkyl 1-acetoxy- $1 H$-indene-2-carboxylates 2a-e and various functionalized- $1 H$-indene esters $\mathbf{3}$ through $\mathrm{S}_{\mathrm{N}}$ 2'-type addition-elimination reaction ${ }^{42}$ as shown in Scheme 2.

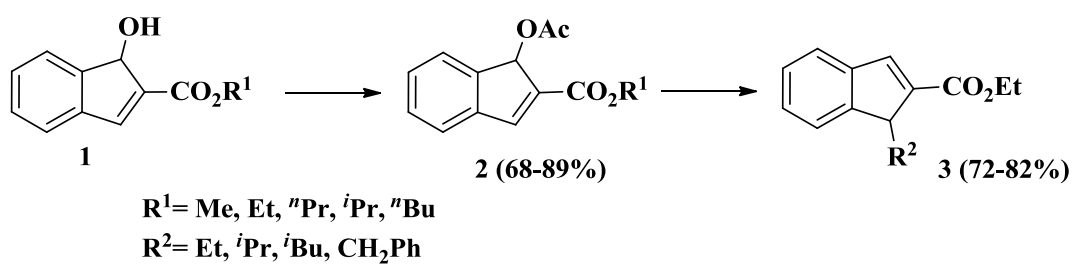

Scheme 2. Synthesis of ethyl- $1 H$-indene-2-carboxylates 3

Continuing with our efforts toward the functionalization of the bicyclic Baylis-Hillman acetate 2 , we report herein the reactivity of the bicyclic Baylis-Hillman adduct $\mathbf{2 b}$ with nitroalkanes salts as nucleophilic reagents. Conjugate addition of nitroalkane to functional allyl acetates has been the subject of intensive work by our group ${ }^{43-47}$. Based on the previous results achieved by our group and others ${ }^{48}$, we further investigated the reactivity of allyl acetates $\mathbf{2 b}$ with nitronate anion generated from primary and secondary nitroalkanes toward the formation of highly substituted ethyl 1 -alkylidene- $1 H$-indene-2carboxylates 4 .
With the aim of developing the best reaction conditions for the coupling of allyl acetate $\mathbf{2} \mathbf{b}$ with nitroethane, several bases have been tested and the representative results are summarized in Table 1 . No products were obtained following the use of 1,8-diazabicyclo[5.4.0] undec-7-ene (DBU) and 1,4-diazabicyclo[2.2.2] octane (DABCO) in THF and $\mathrm{CH}_{3} \mathrm{CN}$ respectively or sodium ethoxide (EtONa) (Table 1, entries 1, 2 and 3). The use of potassium carbonate in THF- $\mathrm{H}_{2} \mathrm{O}$ led to the desired indene $\mathbf{4 a}$ in low yield (23\%, entry 4$)$. The best result was obtained when the allyl acetate $\mathbf{2 b}(2$ mmol) was treated with nitroethane (1.2 equiv.) in $\mathrm{THF}$ at room temperature in the presence of aqueous $\mathrm{NaOH}(0.6 \mathrm{M}, 1.5$ equiv.). The desired compound $4 \mathbf{a}$ was obtained in good yield $(71 \%)$ (Scheme 3).<smiles>CC[C+]COC(=O)C1=Cc2ccccc2C1OC(C)=O</smiles>

$\mathbf{2 b}$

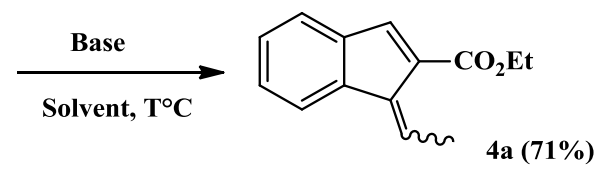

Scheme 3. Synthesis of ethyl 1-ethylidene- $1 H$-indene-2-carboxylate $\mathbf{4 a}$

Table 1. Use of various bases in the synthesis of indene 4a.

\begin{tabular}{cccccc}
\hline Entry & Base & Solvent & $\mathbf{T}^{\circ} \mathbf{C}$ & Time (h) & Yield (\%) \\
\hline 1 & $\mathrm{DBU}$ & $\mathrm{THF}$ & 25 & 3 & - \\
2 & $\mathrm{DABCO}$ & $\mathrm{CH}_{3} \mathrm{CN}$ & 50 & 16 & Mixture \\
3 & $\mathrm{EtONa}$ & $\mathrm{EtOH}$ & 25 & 76 & - \\
4 & $\mathrm{~K}_{2} \mathrm{CO}_{3}$ & $\mathrm{THF}^{-} \mathrm{H}_{2} \mathrm{O}$ & 25 & 24 & 23 \\
5 & $\mathrm{NaOH}(0.6 \mathrm{M})$ & $\mathrm{THF}$ & $0-25$ & 48 & 71 \\
\hline
\end{tabular}

Under the optimized reaction conditions described above, we intended to extend this reaction to a variety of aliphatic nitroalkanes. Different ethyl (E/Z)-1-alkylidene-1H-indene-2carboxylates 4a-e were synthesized in moderate to good yields (Scheme 4, Table 2). The use of secondary nitroalkane (entry 3) under the same reaction conditions, led to the desired compound 4 in low yield (35\%) (Scheme 4). 


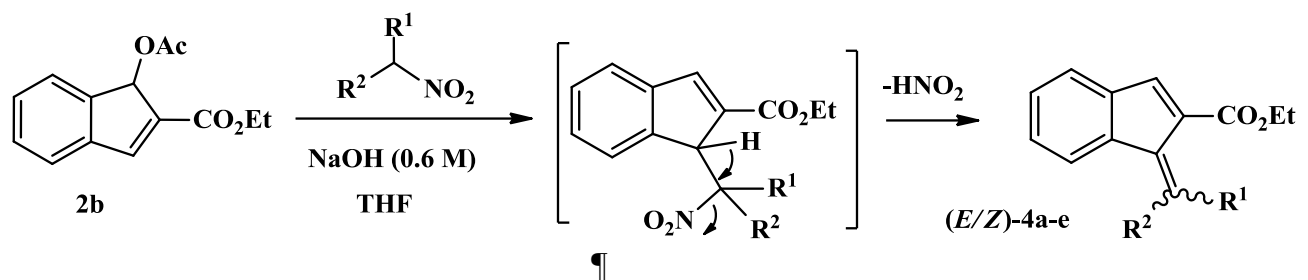

Scheme 4. Synthesis of ethyl (E/Z)-1-alkylidene-1H-indene-2-carboxylate 4

Table 2. Synthesis of ethyl (E/Z)-1-alkylidene-1H-indene-2-carboxylates 4a-e

\begin{tabular}{cccccc}
\hline Indene 4 & $\mathbf{R}^{\mathbf{1}}$ & $\mathbf{R}^{\mathbf{2}}$ & Time (h) & ${\text { Yield }(\boldsymbol{\%})^{\mathbf{a}}}^{(\boldsymbol{E} / \boldsymbol{Z})^{\mathbf{b}}}$ \\
\hline $\mathbf{4 ~ a}$ & $\mathrm{Me}$ & $\mathrm{H}$ & 48 & 71 & $76 / 24$ \\
$\mathbf{4 ~ b}$ & $\mathrm{Et}$ & $\mathrm{H}$ & 48 & 63 & $56 / 44$ \\
$\mathbf{4 ~ c}$ & $\mathrm{Me}$ & $\mathrm{Me}$ & 120 & 35 & - \\
$\mathbf{4 ~ d}$ & ${ }^{n} \mathrm{Pr}$ & $\mathrm{H}$ & 72 & 57 & $70 / 30$ \\
$\mathbf{4 ~ e}$ & ${ }^{n} \mathrm{Bu}$ & $\mathrm{H}$ & 84 & 52 & $62 / 38$ \\
\hline
\end{tabular}

${ }^{a}$ Yields of isolated E/Z indenes 4.

${ }^{b}$ Determined by integration of the exocyclic vinyl proton in ${ }^{l} H N M R$.

Ethyl 1-alkylidene-1H-indene-2-carboxylates 4a-e were obtained as a mixture of two stereoisomers $(E, Z)$. The structures of $\mathbf{4 a - e}$ were established on the basis of their ${ }^{1} \mathrm{H}$ and ${ }^{13} \mathrm{C}$ NMR spectra and by heteronuclear multiple bond correlation (HMBC). Their stereochemistry was clearly assigned on the basis of NOESY experiments.

\section{Conclusion}

In summary, we have developed an efficient two-step protocol for the efficient synthesis of new functionalized ethyl 1-alkylidene- $1 H$-indene-2carboxylates 4 following the coupling reaction of bicyclic Baylis-Hillman acetate $\mathbf{2}$ with nitroalkane salts. The described approach can be explored for the synthesis of new functional indene derivatives as new candidates for biological evaluations, ligand precursors for metallocene catalyst systems and functional materials.

\section{Experimental Section}

All commercially available chemicals and reagents were used without further purification. ${ }^{1} \mathrm{H}-\mathrm{NMR}$ and ${ }^{13} \mathrm{C}$-NMR spectra were recorded on Bruker AMX 300 spectrometer working at $300 \mathrm{MHz}$ and $75 \mathrm{MHz}$ respectively for the proton and ${ }^{13} \mathrm{C}$ with $\mathrm{CDCl}_{3}$ as solvent and TMS as the internal standard. The chemical shifts $(\delta)$ and coupling constants $(J)$ are, respectively, expressed in parts per million (ppm) and Hertz (Hz). All NMR spectra were acquired at room temperature. Assignments of proton $\left({ }^{1} \mathrm{H}-\mathrm{NMR}\right)$ and carbon $\left({ }^{13} \mathrm{C}-\mathrm{NMR}\right)$ signals were secured by DEPT 135, NOESY, HMQC and HMBC experiments. All reactions were monitored by TLC on silica gel plates (Fluka Kieselgel 60 F254, Merck) eluting with the solvents indicated, visualized by a $254 \mathrm{~nm}$ UV lamp and aqueous potassium permanganate solution. For column chromatography, Fluka Kieselgel 70-230 mesh was used. Multiplicity of peaks is indicated by the following: s, singlet; d, doublet; t, triplet; q, quartet; $\mathrm{m}$, multiplet. IR spectra were recorded on an Equinox 55 spectrophotometer. High-Resolution Mass Spectrometry (HRMS) analyses were performed with a Maldi-TOF-TOF technique on a Bruker Autoflex III Smartbeam.

Procedure for the synthesis of alkyl 1-acetoxy-1H-indene-2-carboxylates $2 \mathrm{a}$-e.

To a mixture of alcohol 1 (5 mmol), acetic anhydride ( 25 mmoles) in $40 \mathrm{~mL}$ of anhydrous ether cooled at $0{ }^{\circ} \mathrm{C}$ under stirring in nitrogen atmosphere, was added a drop of concentrated sulfuric acid. After completion of the reaction, the mixture was hydrolyzed with ice water and extracted with ether (3 x $20 \mathrm{~mL}$ ). The organic layers were washed successively with sodium hydroxide solution $(1.5 \mathrm{M})$ and brine until neutral $\mathrm{pH}$ then dried over $\mathrm{MgSO}_{4}$ and concentrated in vacuo. After evaporating of the solvent, the residue obtained was purified on silica gel column chromatography (Hexane/AcOEt, 7/3). (2b)

Ethyl 1-acetoxy-1H-indene-2-carboxylic acid

White solid, yield: $(80 \%), \mathrm{mp} 74-76{ }^{\circ} \mathrm{C}$. IR (ATR): 1708, $1246 \mathrm{~cm}^{-1}$; ${ }^{1} \mathrm{H}-\mathrm{NMR}(300 \mathrm{MHz}$, $\left.\mathrm{CDCl}_{3}\right)$ : 7.67 (s, 1H, Hethylenic); 7.48-7.31 (m, 4H, $H A r) ; 6.67$ (s, $1 \mathrm{H}, \mathrm{CH}) ; 4.28(\mathrm{q}, 2 \mathrm{H}, J=7.5 \mathrm{~Hz}$, $\left.\mathrm{OCH}_{2}\right) ; 2.17\left(\mathrm{~s}, 3 \mathrm{H}, \mathrm{CH}_{3}\right) ; 1.33(\mathrm{t}, 3 \mathrm{H}, J=7.5 \mathrm{~Hz}$, $\left.\mathrm{CH}_{3}\right) ;{ }^{13} \mathrm{C}-\mathrm{NMR}\left(75 \mathrm{MHz}, \mathrm{CDCl}_{3}\right): 170.8(\mathrm{CO})$; 163.2 (CO); 143.8 (=CH); 143.6 (CAr); 140.2 (CAr); 136.7 (=C); 129.3 (CHAr), 129.0 (CHAr), 124.9 (CHAr), 123.8 (CHAr); $74.5(=C H) ; 60.5\left(\mathrm{OCH}_{2}\right)$; $29.7\left(\mathrm{CH}_{3}\right) ; 14.2\left(\mathrm{CH}_{3}\right)$. HRMS calculated for $\mathrm{C}_{14} \mathrm{H}_{14} \mathrm{O}_{4} \mathrm{Na}[\mathrm{M}+\mathrm{Na}]^{+}$269.07843, found 269.07800. 
General procedure for the synthesis of ethyl (E/Z)-1-alkylidene-1H-indene-2-carboxylates 4a-e.

A solution of aqueous $\mathrm{NaOH}(3 \mathrm{mmol}, 0.6 \mathrm{M})$ was added dropwise over a period of $30 \mathrm{~min}$ to an ice-cold solution of allyl acetate $\mathbf{2 b}(2 \mathrm{mmol})$ and nitroalkane $(2.4 \mathrm{mmol})$ in THF $(5 \mathrm{~mL})$. The resulting mixture was stirred at room temperature until total substitution of $\mathbf{2 b}$. The mixture was hydrolyzed with water then extracted with ether $(3 \times 15 \mathrm{~mL})$. The combined organic layers were washed with brine then dried over $\mathrm{MgSO}_{4}$ and concentrated in vacuo. The obtained liquid was purified by flash chromatography (Hexane/AcOEt, $9: 1)$ to provide pure ethyl $(E / Z)$-1-alkylidene- $1 H$ indene carboxylates 4 . The mixture of $E / Z$-indene isomers $\mathbf{4}$ cannot be separated by column chromatography.

Ethyl $(E / Z)$-1-ethylidene-1H-indene-2carboxylate (4a)

Fluorescent yellow oil, yield: $71 \%,{ }^{1} \mathrm{H}-\mathrm{NMR}$ (300 MHz, $\left.\mathrm{CDCl}_{3}\right)$ : 7.78 (s, 1H, Hethylenic); 7.47-7.26 (m, 4H, HAr); 7.67, 7.02 (2q, 1H, $J=7.5$ $\mathrm{Hz}, J=6 \mathrm{~Hz}$, Hethylenic); $4.31(\mathrm{q}, 2 \mathrm{H}, J=7.5 \mathrm{~Hz}$, $\left.\mathrm{OCH}_{2}\right) ; 2.41\left(\mathrm{~d}, 3 \mathrm{H}, J=6 \mathrm{~Hz}, \mathrm{CH}_{3}\right) ; 1.37(\mathrm{t}, 3 \mathrm{H}$, $\left.J=7.5 \mathrm{~Hz}, \mathrm{CH}_{3}\right) ;{ }^{13} \mathrm{C}-\mathrm{NMR}\left(75 \mathrm{MHz}, \mathrm{CDCl}_{3}\right)$ : $164.8(C \mathrm{O}) ; 141.4(=C \mathrm{H}) ; 140.3(=C) ; 139.5(=C \mathrm{H})$; 137.3 (=C); 130.1 (=C); 132.5 (=C); 127.3 (CHAr), 124.4 (CHAr), 122.4 (CHAr), 118.9 (CHAr); 60.1 $\left(\mathrm{OCH}_{2}\right) ; 16.2\left(\mathrm{CH}_{3}\right) ; 14.3\left(\mathrm{CH}_{3}\right)$. HRMS calculated for $\mathrm{C}_{14} \mathrm{H}_{14} \mathrm{O}_{2} \mathrm{Na}[\mathrm{M}+\mathrm{Na}]^{+}$237.0891, found 237.0892.

Ethyl (Z/E)-1-propylidene-1H-indene-2carboxylate (4b)

Fluorescent yellow oil. Yield: 63\%, ${ }^{1} \mathrm{H}-\mathrm{NMR}$ (300 MHz, $\left.\mathrm{CDCl}_{3}\right): 7.82$ (s, 1H, Hethylenic); 7.65-7.25 (m, 4H, HAr); 7.54, $6.87(2 \mathrm{t}, 1 \mathrm{H}, J=9$ $\mathrm{Hz}, J=6 \mathrm{~Hz}$, Hethylenic); $4.31(\mathrm{q}, 2 \mathrm{H}, J=7 \mathrm{~Hz}$, $\left.\mathrm{OCH}_{2}\right) ; 2.85\left(\mathrm{q}, 2 \mathrm{H}, J=7 \mathrm{~Hz}, \mathrm{CH}_{2}\right) ; 1.37(\mathrm{t}, 3 \mathrm{H}$, $\left.J=7 \mathrm{~Hz}, \mathrm{CH}_{3}\right) ; 1.20\left(\mathrm{t}, 3 \mathrm{H}, J=7.5 \mathrm{~Hz}, \mathrm{CH}_{3}\right) ;{ }^{13} \mathrm{C}$ NMR (75 MHz, $\left.\mathrm{CDCl}_{3}\right): 164.7(C \mathrm{O}) ; 141.4(=\mathrm{CH})$; $140.3(=C) ; 138.5(=C H) ; 137.2(=C) ; 136.2(=C)$; 130.1 (=C); 127.7 (CHAr), 127.2 (CHAr), 124.4 (CHAr), 122.5 (CHAr); $60.1\left(\mathrm{OCH}_{2}\right) ; 23.6\left(\mathrm{CH}_{2}\right)$; $14.3\left(\mathrm{CH}_{3}\right) ; 13.7\left(\mathrm{CH}_{3}\right)$. HRMS calculated for $\mathrm{C}_{15} \mathrm{H}_{16} \mathrm{O}_{2} \mathrm{Na}[\mathrm{M}+\mathrm{Na}]^{+}$251.1048, found 251.1050.

Ethyl 1-(propan-2-ylidene)-1H-indene-2carboxylate (4c)

Fluorescent yellow oil. Yield: $35 \%,{ }^{1} \mathrm{H}-\mathrm{NMR}$ (300 MHz, $\left.\mathrm{CDCl}_{3}\right): 7.86$ (s, 1H, Hethylenic);

\section{References}

1- G. Majetich, J. M. Shimkus, J. Nat. Prod. 2010, 73, 284-298.

2- K. Kawazoe, M. Yamamoto, Y. Takaishi, G. Honda, T. Fujita, E. Sezik, E. Yesilada, Phytochemistry, 1999, 50, 493-497.
7.33-7.22 (m, 4H, HAr); 4.25 (q, 2H, $J=7.5 \mathrm{~Hz}$, $\left.\mathrm{OCH}_{2}\right) ; 1.82,1.81\left(2 \mathrm{~s}, 6 \mathrm{H}, 2 \times \mathrm{CH}_{3}\right) ; 1.29(\mathrm{t}, 3 \mathrm{H}$, $\left.J=7.5 \mathrm{~Hz}, \mathrm{CH}_{3}\right) ;{ }^{13} \mathrm{C}-\mathrm{NMR}\left(75 \mathrm{MHz}, \mathrm{CDCl}_{3}\right)$ : 165.0 (CO); $135.8(=C H) ; 134.6(=C) ; 134.1(=C)$; $132.6(=C) ; 131.2 \quad(=C) ; 128.2$ (CHAr), 127.8 (CHAr), 126.3 (CHAr), 125.5 (CHAr); $126.2(=C)$; $61.2\left(\mathrm{OCH}_{2}\right) ; 22.5\left(2 \times \mathrm{CH}_{3}\right) ; 14.2\left(\mathrm{CH}_{3}\right)$.

Ethyl (Z/E)-1-butylidene-1H-indene-2carboxylate $(4 d)$

Fluorescent yellow oil. Yield: 57\%, ${ }^{1} \mathrm{H}-\mathrm{NMR}$ (300 MHz, $\left.\mathrm{CDCl}_{3}\right)$ : 7.79 (s, 1H, Hethylenic); 7.45-7.26 (m, 4H, HAr); 7.57, $6.90(2 \mathrm{t}, 1 \mathrm{H}, J=9$ $\mathrm{Hz}, J=6 \mathrm{~Hz}$, Hethylenic); $4.31(\mathrm{q}, 2 \mathrm{H}, J=7 \mathrm{~Hz}$, $\left.\mathrm{OCH}_{2}\right) ; 2.82\left(\mathrm{q}, 2 \mathrm{H}, J=7 \mathrm{~Hz}, \mathrm{CH}_{2}\right) ; 1.79-1.56(\mathrm{~m}$, $\left.2 \mathrm{H}, \mathrm{CH}_{2}\right) ; 1.37\left(\mathrm{t}, 3 \mathrm{H}, J=7.5 \mathrm{~Hz}, \mathrm{CH}_{3}\right) ; 1.07(\mathrm{t}, 3 \mathrm{H}$, $\left.J=7.5 \mathrm{~Hz}, \mathrm{CH}_{3}\right) ;{ }^{13} \mathrm{C}-\mathrm{NMR}\left(75 \mathrm{MHz}, \mathrm{CDCl}_{3}\right)$ : $164.8(C \mathrm{O}) ; 142.7(=C \mathrm{H}) ; 140.3(=C) ; 138.5(=C \mathrm{H})$; 137.2 (=C); $136.6(=C) ; 129.8$ (=C); 127.6 (CHAr), 127.2 (CHAr), 124.3 (CHAr), 123.2 (CHAr); 60.1 $\left(\mathrm{OCH}_{2}\right) ; 32.3\left(\mathrm{CH}_{2}\right) ; 22.6\left(\mathrm{CH}_{2}\right) ; 14.3\left(\mathrm{CH}_{3}\right) ; 14.1$ $\left(\mathrm{CH}_{3}\right)$. HRMS calculated for $\mathrm{C}_{16} \mathrm{H}_{18} \mathrm{O}_{2} \mathrm{Na}[\mathrm{M}+\mathrm{Na}]^{+}$ 265.1204, found 265.1206.

Ethyl $(Z / E)$-1-pentylidene-1H-indene-2carboxylate (4e)

Fluorescent yellow oil. Yield: 52\%, ${ }^{1} \mathrm{H}-\mathrm{NMR}$ (300 MHz, $\left.\mathrm{CDCl}_{3}\right): 7.80$ (s, 1H, Hethylenic); 7.65-7.27 (m, 4H, HAr); 7.58, $6.90(2 \mathrm{t}, 1 \mathrm{H}, J=9$ $\mathrm{Hz}, J=7.5 \mathrm{~Hz}$, Hethylenic); 4.32 (q, $2 \mathrm{H}, J=7 \mathrm{~Hz}$, $\left.\mathrm{OCH}_{2}\right) ; 2.84$ (q, $\left.2 \mathrm{H}, J=7 \mathrm{~Hz}, \mathrm{CH}_{2}\right) ; 1.70-1.45$ (m, $\left.2 \mathrm{H}, \mathrm{CH}_{2}\right) ; 1.43-1.35\left(\mathrm{~m}, 2 \mathrm{H}, \mathrm{CH}_{2}\right) ; 1.21(\mathrm{t}, 3 \mathrm{H}$, $\left.J=7.5 \mathrm{~Hz}, \mathrm{CH}_{3}\right) ; 0.97\left(\mathrm{t}, 3 \mathrm{H}, J=7.5 \mathrm{~Hz}, \mathrm{CH}_{3}\right)$; ${ }^{13} \mathrm{C}-\mathrm{NMR}\left(75 \mathrm{MHz}, \mathrm{CDCl}_{3}\right)$ : $164.8(\mathrm{CO}) ; 143.0$ $(=C \mathrm{H}) ; 140.2(=C) ; 138.4(=C \mathrm{H}) ; 137.6(=C) ; 136.4$ $(=C) ; 129.9$ (=C); 127.6 (CHAr), 127.2 (CHAr), 124.3 (CHAr), 123.2 (CHAr); $60.1\left(\mathrm{OCH}_{2}\right) ; 31.4$ $\left(\mathrm{CH}_{2}\right) ; 29.7\left(\mathrm{CH}_{2}\right) ; 22.5\left(\mathrm{CH}_{2}\right) ; 14.3\left(\mathrm{CH}_{3}\right) ; 14.1$ $\left(\mathrm{CH}_{3}\right)$. HRMS calculated for $\mathrm{C}_{17} \mathrm{H}_{20} \mathrm{O}_{2} \mathrm{Na}[\mathrm{M}+\mathrm{Na}]^{+}$ 279.1361, found 279.1363.

\section{Acknowledgements}

This work was supported by the Ministry of Higher Education and Scientific Research of Tunisia. The authors wish to thank professor Jacques Lebreton (University of Nantes, France) for carrying out HRMS analyzes and logistical help.

3- A. N. Nicholson, P. A. Pascoe, C. Turner, C. R. Ganellin, P. M. Greengrass, A. F. Casy, A. D. Mercer, Br. J. Pharmacol., 1991, 104, 270-276.

4- T. Koike, Y. Hoashi, T. Takai, M. Nakayama, N. Yukuhiro, T. Ishikawa, K. Hirai, O. Uchikawa, J. Med. Chem., 2011, 54, 3436-3444. 
5- R. Kolanos, U. Siripurapu, M. Pullagurla, M. Riaz, V. Setola, B. L. Roth, M. Dukat, R. A. Glennon, Bioorg. Med. Chem. Lett., 2005, 15, 1987-1991.

6- T. Oda, Y. So, Y. Sato, N. Shimizu, H. Handa, Y. Yasukochi, T. Kasahara, Mutat. Res., 2003, 534, 187-195.

7- M. R. Schneider, H. Ball, J. Med. Chem., 1986, 29, 75-79.

8- S. H. Mehdi, R. Hashim, R. M. Ghalib, M. F. C. Guedes da Silva, O. Sulaiman, S. Z. Rahman, V. Murugaiyah, M. M. Marimuthu, J. Mol. Struct., 2011, 1006, 318-323.

9- A. Korte, J. Legros, C. Bolm, Synlett 2004, 2397-2399.

10- T. Katoh, T. Akagi, C. Noguchi, T. Kajimoto, M. Node, R. Tanaka, M. Nishizawa, H. Ohtsu, N. Suzuki, K. Saito, Bioorg. Med. Chem. 2007, 15, 2736-2748.

11- P.-C. Lee, H.J. Lee, R. Kakadiya, K. Sanjiv, T.-L. Su, T.C. Lee, Oncogene, 2013, 32, 1144-1154.

12- N. Watanabe, H. Nakagawa, A. Ikeno, H. Minato, C. Kohayakawa, J.I. Tsuji, Bioorg. Med. Chem. Lett., 2003, 13, 4317-4320.

13- K. Serge, G. K. Pantelis, P. Daniel, B. Francoise, V. D. V. Patrick, II Farmaco 1999, 54, 678-683.

14- X. Zhu, H. Tsuji, K. Nakabayashi, S.I. Ohkoshi, E. Nakamura, J. Am. Chem. Soc., 2011, 133, 16342-16345.

15- H. G. Alt, A. Köppl, Chem. Rev. 2000, 100, 1205-1222.

16- C. Li, Y. Zeng, J. Wang, Tetrahedron Lett., 2009, 50, 2956-2959.

17- H. Kheira, P. Li, J. Xu, J. Mol. Cat. A: Chem., 2014, 391, 168-174.

18- E. J. Park, S. H. Kim, S. Chang, J. Am. Chem. Soc., 2008, 130, 17268-17269.

19- G. B. Bajracharya, N. K. Pahadi, I. D. Gridnev, Y. Yamamoto, J. Org. Chem.,2006, 71, 6204-6210.

20- T. Takahashi, Y. Kuzuba, F. Kong, K. Nakajima, Z. Xi, J. Am. Chem. Soc., 2005, 127, 17188-17189.

21- K. Bahadur, S. Magar, Y. R. Lee, Org. Lett., 2013, 15, 4288-4291.

22- E. M. Coyanis, J. L. Panayides, M. A. Fernandes, C. B. Koning, A. L. Willem, J. Organomet. Chem., 2006, 691, 5222-5239.

23- X. Zhang, W. T. Teo, W. Rao, D.-L. Ma, C.H. Leung, P. W. H. Chan, Tetrahedron Lett., 2014, 55, 3881-3884.

24- J. Shao, P. Hu, G. Hong, M. Fang, X. Li, X. Xu, Synlett 2014, 25, 1009-1013.

25- D. Basavaiah, B. S. Reddy, H. Lingam Tetrahedron, 2013, 69, 1994-2003.
26- D. Eom, S. Park, Y. Park, T. Ryu, P. H. Lee, Org. Lett., 2012, 14, 5392-5395.

27- H. Kurouchi, H. Sugimoto, Y. Otani, T. Ohwada, J. Am. Chem. Soc., 2010, 132, 807-815.

28- Z. A. Khan, T. Wirth, Org. Lett, 2009, 11, 229-231.

29- C. D. Smith, G. Rosocha, L. Mui, R. A. Batey, J. Org. Chem., 2010, 75, 4716-4727.

30- X. Zhou, H. Zhang, X. Xie, Y. Li, J. Org. Chem. 2008, 73, 3958-3960.

31- Y. Kuninobu, Y. Nishina, M. Shouho, K. Takai, Angew. Chem. Int. Ed., 2006, 45, 2766-2768.

32- P. G. Gassman, J. A. Ray, P. G. Wenthold, J. Mickelson, W. J. Org. Chem., 1991, 56, 5143-5146.

33- Q. Hou, Z. Zhang, F. Kong, S. Wang, H. Wang, Z.-J. Yao, Chem. Commun., 2013, 49, 695-697.

34- X. Shi, C. Li, Org. Lett., 2013, 15, 1476-1479.

35- J. Zhao, D. A. Clark, Org. Lett., 2012, 14, 1668-1671.

36- C. S. Bryan, M. Lautens, Org. Lett., 2010 , 12, 2754-2757.

37- T. Furuta, T. Asakawa, M. Iinuma, S. Fujii, K. Tnaka, T. Kan, Chem. Commun., 2006, 3648-3650.

38- F. W. Patureau, T. Besset, N. Kuhl, F. Glorius, J. Am. Chem. Soc., 2011, 133, 2154-2156.

39- S. Taktouk, J. Ben Kraïem, H. Chaabane, J. Lebreton, H. Amri, Med. J. Chem., 2013, 2, 658-666.

40- A. R. Daniewski, J. Kiegiel, J. Org. Chem., 1988, 53, 5535-5538.

41- K. Samula, B. Cichy, Acta Pol. Pharm., 1985, 42, 256; Chem. Abstr., 1986, 105, $171931 \mathrm{v}$.

42- S. Taktouk, J. Ben Kraïem, H. Amri, Synth. Commun., 2013, 44, 2004-2011.

43- A. Chamakh, M. M'hirsi, J. Villieras, J. Lebreton, H. Amri, Synthesis, 2000, 295-299.

44- J. Ben Kraïem, H. Amri, J. Soc. Chim.Tunisie, 2011, 13, 35-39.

45- H. Kraïem, T. Turki, H. Amri, Synth. Commun., 2003, 33, 3261-3269.

46- F. Beji, J. Lebreton, J. Villiéras, H. Amri, Tetrahedron, 2001, 57, 9959-9962.

47- A. Fray, J. Ben Kraïem, H. Amri, Med. J. Chem., 2014, 3, 907-915.

48- I. Yavari, L. Moradi, A. MokhtarporyaniSanandej, A. Mirzaei, Helv. Chim. Acta, 2007, 90, 392-394. 